\title{
Coordination of sucrose uptake and respiration in the yeast Debaryomyces yamadae
}

\author{
Janko Kaliterna, † Ruud A. Weusthuis, $\ddagger$ Juan I. Castrillo, \\ Johannes P. van Dijken and Jack T. Pronk
}

Author for correspondence: Jack T. Pronk. Tel: +31 1578 2387. Fax: +31 15782355.

Department of Microbiology and Enzymology, Kluyver Laboratory of

Biotechnology, Delft University of Technology, Julianalaan $67,2628 \mathrm{BC}$ Delft, The Netherlands

Screening in batch cultures identified Debaryomyces yamadae as a yeast that exhibits the Kluyver effect for sucrose: this disaccharide can be respired but, even under oxygen-limited conditions, alcoholic fermentation of sucrose does not occur. Ethanol, glycerol and arabitol were the main fermentation products during oxygen-limited growth on glucose in chemostat cultures. None of these fermentation products were produced in oxygen-limited chemostat cultures grown on sucrose and the fraction of the sucrose that could not be respired remained unused in the culture medium. This absence of alcoholic fermentation was not due to repression of the key fermentative enzymes pyruvate decarboxylase and alcohol dehydrogenase. In contrast to some other yeasts that exhibit a Kluyver effect, $D$. yamadae did not exhibit a preference for ethanol in batch cultures grown on mixtures of ethanol and sucrose. Sucrose metabolism in $D$. yamadae involves intracellular hydrolysis by an $\alpha$-glucosidase. Incubation of weakly buffered cell suspensions with sucrose led to a rapid transient alkalinization, indicating the presence of a sucrose-proton symport system. The apparent substrate saturation constant of the sucroseuptake system was $0.2 \mathrm{mmol} \mathrm{I}^{-1}$. Sucrose-dependent alkalinization rates were much lower in samples from oxygen-limited cultures than in samples from aerobic cultures. Transient responses of $D$. yamadae to oxygen limitation were investigated by applying a sudden decrease in the oxygen feed to aerobic sugar-limited chemostat cultures. In glucose-grown cultures, this led to alcoholic fermentation and no significant accumulation of sugar occurred after the switch. In sucrose-limited cultures, sugar accumulation occurred instantaneously after the switch, and ethanol formation was virtually absent. The results indicate that the Kluyver effect for sucrose in D. yamadae, i.e. the adjustment of the glycolytic flux to the cells' respiratory capacity, is effected by rapid down-regulation of the capacity of the sucrose carrier under oxygenlimited conditions.

Keywords: alcoholic fermentation, Kluyver effect, sucrose metabolism, invertase, transport

\section{INTRODUCTION}

The Kluyver effect is a widespread phenomenon among facultatively fermentative yeasts. Yeasts that exhibit the Kluyver effect are unable to ferment certain disaccharides to ethanol and $\mathrm{CO}_{2}$, even though respiratory metabolism of these disaccharides, and alcoholic fermentation of the component hexose(s) are possible (Sims \& Barnett, 1978; Weusthuis et al., 1994a, b).

†Present address: PLIVA d.d., Research Institute, Zagreb, Croatia.

$\ddagger$ Present address: ATO-DLO, Wageningen, The Netherlands.
We have recently studied the Kluyver effect for maltose in the yeast Candida utilis in steady-state and transient-state chemostat cultures at various oxygen feeds (Weusthuis $e t$ al., 1994a, b; Kaliterna et al., 1995). In oxygen-limited, steady-state chemostat cultures grown on maltose, the rate of disaccharide metabolism was tightly adjusted to the cultures' respiratory activity which, under oxygenlimited conditions, was determined by electron-acceptor availability. As a consequence, alcoholic fermentation did not occur and the fraction of the disaccharide that could not be respired remained unconsumed (Weusthuis et al., 1994a, b). However, when an aerobic, maltose-limited 
culture of $C$. utilis was suddenly exposed to oxygen limitation, a transient accumulation of ethanol occurred (Kaliterna et al., 1995). The kinetics of this transient alcoholic fermentation were consistent with regulation of a key step in disaccharide metabolism, probably disaccharide transport, at the level of enzyme synthesis (Kaliterna et al., 1995). In batch cultures of $C$. utilis grown on mixtures of maltose and ethanol, the latter compound was the preferred substrate, suggesting that ethanol or a related metabolite might be the effector molecule in this regulatory process (Weusthuis et al., 1994b).

At present, it is unclear to what extent the observations made with $C$. utilis are representative for other 'Kluyverpositive' yeasts and disaccharides. A particularly interesting case in this respect is the fact that many yeasts exhibit a Kluyver effect for sucrose. According to the literature, the Kluyver effect for sucrose is widespread: of 137 facultatively fermentative sucrose-utilizing yeasts, 31 were reported to be unable to ferment this sugar (Barnett et al., 1990). In many yeasts, including Saccharomyces cerevisiae, sucrose metabolism is initiated by extracellular hydrolysis, catalysed by invertase, followed by uptake of the monosaccharides. Clearly, the occurrence of such a system in a yeast that exhibits a Kluyver effect for sucrose would rule out a regulation of sucrose metabolism at the level of disaccharide transport. Both for this reason and in view of the observed substrate-specificities of yeasts with a Kluyver effect for sucrose, it has been postulated that occurrence of a Kluyver effect for this sugar should be accompanied by its intracellular hydrolysis (Sims \& Barnett, 1978; Barnett, 1981). However, this possibility has not been studied experimentally.

The aim of this work was to test the hypothesis that sucrose hydrolysis in a yeast with a Kluyver effect for this disaccharide occurs intracellularly and to investigate to what extent regulation of disaccharide metabolism is comparable with that of maltose metabolism in C. utilis. Debaryomyces yamadae CBS 7035 , a yeast that has previously been reported to exhibit a Kluyver effect for sucrose (Barnett et al., 1990), was selected as the model organism.

\section{METHODS}

Yeast strains and maintenance. Candida kruisii CBS 6451, Candida naeodendra CBS 6032, Candida peltata CBS 5564, Candida shehatae CBS 5813, Debaryomyces yamadae CBS 7035, Metschnikowia reukaufii CBS 611, Hansenula polymorpha CBS 2575 and Pichia stipitis CBS 5773 were obtained from the Centraalbureau voor Schimmelcultures (Delft, The Netherlands) and maintained on malt agar slants at $4{ }^{\circ} \mathrm{C}$. These strains have all been reported to grow on sucrose aerobically, but not to ferment this disaccharide (Barnett et al., 1990).

Screening for the Kluyver effect in shake-flask cultures. The ability to ferment glucose and sucrose was investigated in $100 \mathrm{ml}$ shake-flask cultures containing $50 \mathrm{ml}$ mineral medium (Van Leeuwen et al., 1992) supplemented with either $20 \mathrm{~g}$ glucose $\mathrm{l}^{-1}$ or $20 \mathrm{~g}$ sucrose $\mathrm{I}^{-1}$. After $24 \mathrm{~h}$ incubation at $30^{\circ} \mathrm{C}$ on a rotatory shaker at 200 r.p.m., samples were withdrawn and analysed for biomass dry weight and ethanol.

Chemostat cultivation. Chemostat cultivation of $D$. yamadae CBS 7035 was performed in 21 fermenters (Applikon) at a dilution rate of $0.10 \mathrm{~h}^{-1}$, a temperature of $30^{\circ} \mathrm{C}$ and a stirrer speed of 1050 r.p.m. The culture $\mathrm{pH}$ was maintained at 5.0 by automatic addition of $2 \mathrm{M} \mathrm{KOH}$ via an Applikon ADI-1020 biocontroller. Aerobic cultivation (dissolved oxygen concentration above $70 \%$ of air saturation) was performed by maintaining an airflow through the culture of $0.531 \mathrm{~min}^{-1}$. This was administered to the culture by two means: $0.51 \mathrm{~min}^{-1}$ with a Brooks 5876 mass-flow controller and $30 \mathrm{ml} \mathrm{min}^{-1}$ with a peristaltic pump. The working volume of the culture was kept at $1.0 \mathrm{l}$ by removal of effluent from below the surface of the culture, via an electrical level controller. This set-up ensured that biomass concentrations in the effluent differed by less than $1 \%$ from those in samples taken directly from the culture (Noorman et al., 1991). In order to obtain reproducible gastransfer properties, positions of baffles, pipes, impellers and sensors were identical in the different fermenters used in this study. To avoid loss of volatile metabolites, the off-gas condenser was cooled to $2{ }^{\circ} \mathrm{C}$. The mineral medium was prepared according to Van Leeuwen et al. (1992). Sucrose $\left(10 \mathrm{~g} \mathrm{l}^{-1}\right)$ or glucose $\left(10 \mathrm{~g} \mathrm{l}^{-1}\right)$ and vitamins were added to the cultures after separate sterilization. After sterilization, approximately $1 \%(\mathrm{w} / \mathrm{w})$ of the sucrose was found to be hydrolysed to glucose and fructose. Culture purity was routinely checked by phase-contrast microscopy at $1000 \times$ magnification.

Transient-state experiments. At zero time, the gas flow through the mass-flow controller was switched from air to nitrogen gas, resulting in the addition of a mixture of ultra-pure nitrogen gas $\left(0.5 \mathrm{l} \mathrm{min}^{-1}\right)$ and air $\left(30 \mathrm{ml} \mathrm{min}^{-1}\right)$ to the culture. As a result, the dissolved-oxygen concentration in the culture, measured with an Ingold polarographic oxygen electrode, decreased to below $0.1 \%$ of air saturation within $2 \mathrm{~min}$. At appropriate intervals, samples from the effluent line were collected on ice. The residence time in the effluent line was approximately $1 \mathrm{~min}$. Samples were analysed for culture dry weight and, after centrifugation at $10000 \mathrm{~g}$, for metabolites. Cell pellets for preparation of cell-free extracts were resuspended in $10 \mathrm{mM}$ potassium phosphate buffer ( $\mathrm{pH} \mathrm{7.5)} \mathrm{containing} 2 \mathrm{mM}$ EDTA, frozen and stored at $-20^{\circ} \mathrm{C}$ (Postma et al., 1989).

Batch cultivation. Yeasts were pregrown in $100 \mathrm{ml}$ shake-flasks on $20 \mathrm{ml}$ of the mineral medium described above, with an initial $\mathrm{pH}$ of 6.0 and containing $5.0 \mathrm{~g}$ glucose or sucrose $\mathrm{l}^{-1}$. Batch cultivation took place in the fermenters described above with an initial working volume of $1.0 \mathrm{l}$. The medium was the same as used for continuous cultivation with $5 \mathrm{~g}$ glucose or sucrose $\mathrm{l}^{-1}$ and $4 \mathrm{~g}$ ethanol $\mathrm{l}^{-1}$. The $\mathrm{pH}$ was controlled between $4 \cdot 9$ and $5 \cdot 1$ by the automatic addition of $2 \mathrm{M} \mathrm{KOH}$ or $1 \mathrm{M} \mathrm{H}_{2} \mathrm{SO}_{4}$. The airflow rate into the culture was $1.01 \mathrm{~min}^{-1}$. The dissolved oxygen concentration in the culture was kept above $50 \%$ air saturation by manual adjustment of the stirring speed between 750 and 1250 r.p.m. The temperature was $30^{\circ} \mathrm{C}$.

Metabolite analysis. Enzymic analysis of glucose concentrations and HPLC analysis of ethanol, glycerol, lactate and other low-molecular-mass metabolites was performed as described by Weusthuis $e$ t al. (1993). Ethanol was also determined with an enzymic assay (based on alcohol oxidase, EK 001, Leeds Biochemicals). This method gave the same results, but has a lower detection limit (approximately $10 \mu \mathrm{M}$ ) than the HPLC method (approximately $0.2 \mathrm{mM}$ ). Sucrose was determined via hydrolysis to glucose and fructose in $32 \mathrm{mM}$ sodium citrate buffer ( $\mathrm{pH} \mathrm{4.6)}$ with $50 \mathrm{U}$ invertase $\mathrm{ml}^{-1}$, followed by enzymic glucose analysis.

Biomass determination. Dry weights of culture samples were determined via filtration with $0.45 \mu \mathrm{m}$ membrane filters and drying in a microwave oven (Postma et al., 1989). Parallel samples varied by less than $1 \%$. $O D_{660}$ was measured with a Vita-Lab 20 (Vita Scientific). When the $\mathrm{OD}_{600}$ was above $0 \cdot 3$, samples were diluted with demineralized water. 
Preparation of cell-free extracts. Cell-free extracts of chemostat-grown cells were prepared as described by Postma et al. (1989).

Pyruvate decarboxylase and alcohol dehydrogenase assays. Assays of pyruvate decarboxylase (EC 4.1.1.1) and alcohol dehydrogenase (EC 1.1.1.1) activity in cell-free extracts were performed as described by Postma et al. (1989).

Sucrose-hydrolase assays. Sucrose-hydrolysing activity was measured in culture supernatants, cell-free extracts and washed cell suspensions. The latter were obtained by washing culture samples twice with $50 \mathrm{mM}$ sodium acetate buffer (pH 5). Sucrose-hydrolysing activities of the various preparations were assayed at $30^{\circ} \mathrm{C}$ by following the rate of glucose production in an incubation mixture containing $50 \mathrm{mM}$ sodium acetate buffer $(\mathrm{pH} 5.0)$ and $56 \mathrm{mM}$ sucrose. The reaction was stopped by the addition of Tris buffer $(\mathrm{pH} 10 \cdot 5)$ to a final concentration of $60 \mathrm{mM}$. When assays were performed at $\mathrm{pH} 7 \cdot 0$, the sodium acetate buffer was replaced by $50 \mathrm{mM}$ potassium phosphate buffer. For substrate-specificity assays, maltose or raffinose $(56 \mathrm{mM})$ were used instead of sucrose. In the case of raffinose, production of fructose was determined with a commercial kit (Boehringer no. 139 106). One unit is defined as the hydrolysis of $1 \mu \mathrm{mol}$ disaccharide $\mathrm{min}^{-1}$.

Sucrose-proton symport assays Sucrose transport was determined by measuring initial rates of sucrose-dependent alkalinization of weakly buffered cell suspensions according to the method of van Urk et al. (1989). To calculate proton-uptake rates, alkalinization curves were calibrated with a standard $\mathrm{NaOH}$ solution. Kinetic parameters were estimated by nonlinear regression, assuming Michaelis-Menten kinetics, using the program Fig.P (Fig.P Software).

\section{RESULTS}

\section{Choice of model organism}

In taxonomic studies, occurrence of alcoholic fermentation is generally tested with the Durham-tube method. Although this method allows rapid screening for vigorous fermentation of substrates, low rates of alcoholic fermentation easily go unnoticed (van Dijken et al., 1986; Barnett, 1992). For physiological studies on the Kluyver effect, it is desirable to use strains with a clear-cut phenotype. Therefore, eight yeast strains previously reported to grow on sucrose aerobically, but not to ferment this disaccharide (Barnett et al., 1990), were tested by enzymic analysis of ethanol formation in shake-flask cultures (Table 1). This method is based on the fact that shake-flask cultures of yeasts almost inevitably become oxygen limited (van Dijken et al., 1986), which induces ethanol formation from fermentable sugars.

Surprisingly, four out of the eight yeast strains tested produced significant amounts of ethanol in sucrose-grown shake-flask cultures (Table 1). A fifth yeast, M. reukaufii, grew extremely slowly under the experimental conditions and was therefore rejected as a model organism. C. peltata, which did not exhibit alcoholic fermentation on sucrose, was not chosen because it produced large amounts of a viscous extracellular product. This left two strains which fermented glucose, but not sucrose: C. naeodendra and $D$. yamadae. The latter was chosen for further studies because it exhibited the highest ethanol concentration in glucosegrown cultures (Table 1).

\section{Glucose and sucrose metabolism in steady-state chemostat cultures}

To further investigate the Kluyver effect for sucrose in $D$. yamadae CBS 7035, the organism was grown on glucose and sucrose in aerobic and oxygen-limited chemostat cultures $\left(D=0 \cdot 10 \mathrm{~h}^{-1}\right)$.

In aerobic, sugar-limited chemostat cultures grown on glucose or sucrose, all sugar entering the fermenter could be recovered as biomass and $\mathrm{CO}_{2}$ and no fermentation

Table 1. Biomass and ethanol concentrations in $24 \mathrm{~h}$ oxygen-limited shake-flask cultures on glucose and sucrose of various facultatively fermentative yeast strains with reported Kluyver effects for sucrose

\begin{tabular}{|c|c|c|c|c|}
\hline \multirow[t]{2}{*}{ Yeast strain } & \multicolumn{2}{|c|}{ Glucose-grown cultures } & \multicolumn{2}{|c|}{ Sucrose-grown cultures } \\
\hline & $\begin{array}{c}\text { Biomass } \\
\left(\mathrm{g} \mathrm{l}^{-1}\right)\end{array}$ & $\begin{array}{c}\text { Ethanol } \\
\left(\mathrm{g}^{-1}\right)\end{array}$ & $\begin{array}{c}\text { Biomass } \\
\left(\mathrm{g}^{-1}\right)\end{array}$ & $\begin{array}{c}\text { Ethanol } \\
\left(\mathrm{g} \mathrm{l}^{-1}\right)\end{array}$ \\
\hline Candida kruisii CBS 6451 & $2 \cdot 7$ & $5 \cdot 8$ & $2 \cdot 7$ & $1 \cdot 3$ \\
\hline Candida naeodendra CBS 6032 & $5 \cdot 8$ & 0.9 & $1 \cdot 7$ & $<0.01$ \\
\hline Candida peltata CBS 5564* & - & $6 \cdot 2$ & - & $<0.01$ \\
\hline Candida shehatae CBS 5813 & $2 \cdot 4$ & $5 \cdot 0$ & $2 \cdot 6$ & $0 \cdot 2$ \\
\hline Debaryomyces yamadae CBS 7035 & $2 \cdot 8$ & $5 \cdot 3$ & $2 \cdot 5$ & $<0.01$ \\
\hline Metschnikowia reukaufii CBS 611 & ND & ND & ND & $0 \cdot 02$ \\
\hline Hansenula polymorpha CBS 2575 & ND & ND & ND & 1.6 \\
\hline Pichia stipitis CBS 5773 & ND & ND & ND & $1 \cdot 5$ \\
\hline
\end{tabular}

ND, Not determined.

${ }^{*}$ C. peltata CBS 5564 formed a viscous extracellular product, which prevented the measurement of culture dry weights by filtration. 
Table 2. Concentrations of substrate, metabolites and biomass in carbon- and oxygenlimited chemostat cultures of $D$. yamadae grown on glucose or sucrose $\left(D=0.10 \mathrm{~h}^{-1}\right.$, reservoir concentration $10 \mathrm{~g}_{\text {sugar }} \mathrm{I}^{-1}$ )

All metabolite concentrations are given in $\mathrm{mmol}^{-1}$. The experiments were performed in triplicate; data are from representative steady-state cultures.

\begin{tabular}{|c|c|c|c|c|}
\hline & \multicolumn{2}{|c|}{ Glucose-grown cultures } & \multicolumn{2}{|c|}{ Sucrose-grown cultures } \\
\hline & Aerobic & $\mathrm{O}_{2}$ limited & Aerobic & $\mathbf{O}_{2}$ limited \\
\hline Sucrose (mM) & $<0 \cdot 1$ & $<0 \cdot 1$ & $<0 \cdot 1$ & $23 \cdot 3$ \\
\hline Glucose $(\mathrm{mM})$ & $<0 \cdot 1$ & $<0 \cdot 1$ & $<0 \cdot 1$ & 0.65 \\
\hline Ethanol (mM) & $<0 \cdot 1$ & $29 \cdot 9$ & $<0 \cdot 1$ & $<0 \cdot 1$ \\
\hline Glycerol (mM) & $<0 \cdot 1$ & $10 \cdot 4$ & $<0 \cdot 1$ & $<0 \cdot 1$ \\
\hline Arabitol (mM) & $<0.1$ & $1 \cdot 2$ & $<0 \cdot 1$ & $<0 \cdot 1$ \\
\hline Biomass $\left(\mathrm{g} \mathrm{l}^{-1}\right)$ & $4 \cdot 9$ & $2 \cdot 2$ & $5 \cdot 1$ & $1 \cdot 4$ \\
\hline
\end{tabular}

products were detected in culture supernatants (Table 2). The biomass yield on the growth-limiting sugar was $0.5 \mathrm{~g}$ biomass $(\mathrm{g} \text { sugar })^{-1}$. In oxygen-limited chemostat cultures grown on glucose, a mixed fermentative metabolism was observed. In addition to ethanol, significant amounts of glycerol and arabitol were produced (Table 2; the latter compound was tentatively identified, based on its retention time on HPLC). No residual glucose was detected in the oxygen-limited steady-state cultures and carbon recoveries were $95-97 \%$. The respiro-fermentative metabolism led to a decrease of the biomass yield to approximately $0.2 \mathrm{~g}$ biomass (g glucose) $)^{-1}$.

In sucrose-grown oxygen-limited cultures of D. yamadae, the substrate was only partially consumed and the fermentation products observed in the glucose-grown oxygen-limited cultures were not detected (Table 2). Apparently, the mechanism responsible for the Kluyver effect in this yeast not only prevents alcoholic fermentation, but also the formation of other fermentation products. The biomass yield on sucrose, corrected for the high residual sugar concentration, was $0.5 \mathrm{~g}$ biomass ( $\mathrm{g}$ sucrose $)^{-1}$, consistent with a fully respiratory metabolism.

\section{Localization of the sucrose-hydrolysing activity}

Disaccharide uptake has been implicated as a key process in the regulation of sugar metabolism in yeasts which exhibit a Kluyver effect (Sims \& Barnett, 1978; Barnett, 1981, 1992; Kaliterna et al., 1995). Since this can only hold when disaccharide hydrolysis occurs intracellularly, the localization of the sucrose-hydrolysing enzyme activity in D. yamadae was investigated.

Neither in aerobic, sucrose-limited nor in oxygen-limited chemostat cultures of $D$. yamadae could sucrosehydrolysing activity be detected in culture supernatants. This observation does not necessarily rule out the possibility of extracytosolic sucrose hydrolysis since invertase can also be retained in the yeast cell wall (Barnett, 1981). However, washed cell suspensions also did not exhibit significant sucrose-hydrolysing activity. The same results were obtained when transport and phosphorylation of sugars were inhibited by the addition of $0.1 \mathrm{mM}$ 2,4-dinitrophenol. These observations argue against the presence of cell-wall-associated invertase and suggest that, instead, sucrose is hydrolysed intracellularly.

Indeed, cell-free extracts of aerobic, sucrose-limited chemostat cultures exhibited high sucrose-hydrolysing activities. Activity at $\mathrm{pH} 7\left[1.8 \mathrm{U}(\mathrm{mg} \text { protein })^{-1}\right]$ was $2 \cdot 4$ fold higher than at $\mathrm{pH} 5$, consistent with a cytosolic localization (Barnett, 1981). Sucrose-hydrolysing activities of extracts from oxygen-limited cultures were only approximately $30 \%$ lower than those in aerobic, sucroselimited cultures $[1.2 \mathrm{U} \text { (mg protein })^{-1}$, indicating that the inability to utilize all sucrose in the oxygen-limited cultures was not due to repression of the hydrolase.

In theory, intracellular hydrolysis of sucrose can be catalysed either by a $\beta$-fructosidase (invertase; EC 3.2 .1 .26 ) or by an $\alpha$-glucosidase ('maltase', EC 3.2.1.20) (Barnett, 1981). One way to discriminate between these two possibilities is to investigate the substrate specificity of the sucrose-hydrolysing enzyme activities: $\beta$-fructosidase hydrolyses sucrose and raffinose, but not maltose; $\alpha$-glucosidase hydrolyses maltose and sucrose, but not raffinose (Barnett, 1981). Rates of maltose and sucrose hydrolysis by cell-free extracts of $D$. yamadae grown under either aerobic or oxygen-limited conditions differed by less than $10 \%$ (data not shown). Raffinose was not hydrolysed, indicating that sucrose hydrolysis in this yeast is catalysed by an intracellular $\alpha$-glucosidase.

\section{Sucrose transport}

Since sucrose cannot cross the yeast plasma membrane by passive diffusion, its metabolism via an intracellular $\alpha$ glucosidase requires the presence of a sucrose carrier. Disaccharide transport in yeasts generally takes place via proton-symport (Serrano, 1977; Schulz \& Höfer, 1986; Santos et al., 1982; Dickson \& Barr, 1983; Carvalho-Silva \& Spencer-Martins, 1990).

Addition of sucrose to weakly buffered cell suspensions of D. yamadae, pregrown in aerobic, sucrose-limited chemo- 

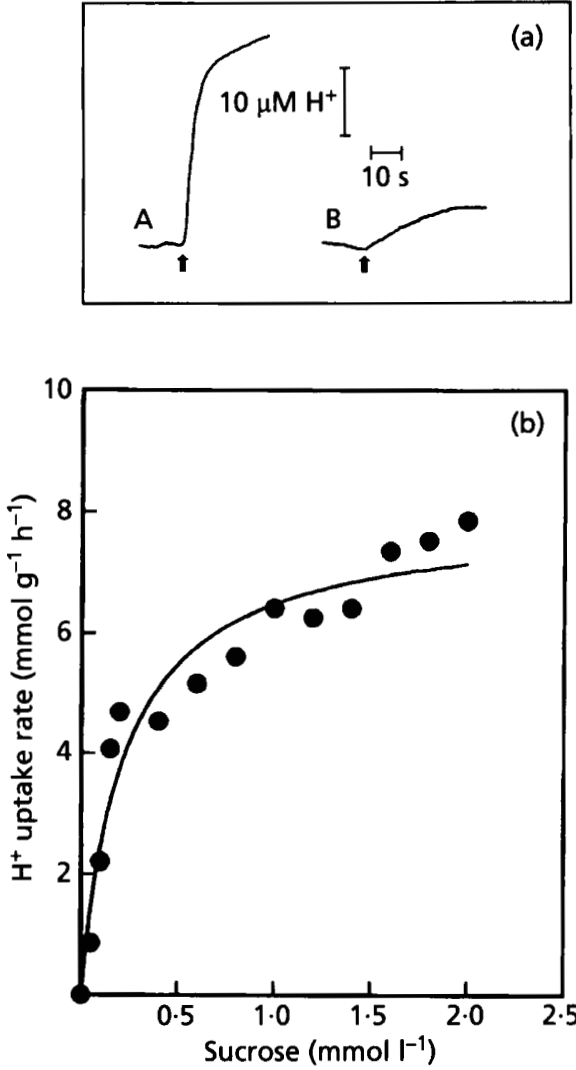

Fig. 1. (a) Sugar-dependent effects on the $\mathrm{pH}$ of weakly buffered cell suspensions of $D$. yamadae CBS 7035 grown on sucrose in aerobic (A) or oxygen-limited chemostat cultures (B). At the time indicated by the arrow, $20 \mathrm{mM}$ sucrose was added. An identical biomass concentration $\left(2.0 \mathrm{~g}\right.$ dry $\left.w \mathrm{I}^{-1}\right)$ was used in both experiments. (b) Kinetics of sucrose-dependent alkalinization of weakly buffered cell suspensions of $D$. yamadae pregrown in aerobic, sucrose-limited chemostat culture. The line drawn through the points was calculated by non-linear regression, assuming Michaelis-Menten kinetics.

stat cultures, led to a rapid transient alkalinization (Fig. 1a), which is indicative of the presence of a sucroseproton symporter in this yeast. Uptake of sucrose by aerobically grown cells showed saturation kinetics (Fig. 1b) and non-linear regression analysis of the uptake activities revealed a $K_{\mathrm{m}}$ for sucrose uptake of $0.22 \pm 0.05 \mathrm{mmol}$ sucrose $1^{-1}$ and a $V_{\max }$ of $7.9 \mathrm{mmol} \mathrm{H}^{+} \mathrm{g}^{-1} \mathrm{~h}^{-1}$.

Assuming that, as has been observed for other yeast sugar-proton symporters (Williamson et al., 1993; Weusthuis et al., 1993), the proton-sugar stoichiometry is near unity, the capacity of this uptake system is 14-fold higher than the in situ rate of sucrose consumption in the aerobic, sucrose-limited chemostat cultures (0.55 mmol g $\mathrm{m}^{-1} \mathrm{~h}^{-1}$; calculated from Table 2).

If coordination of the glycolytic flux and the cells' respiratory capacity is indeed regulated at the level of sucrose uptake, this would require a drastic reduction in the capacity of the sucrose carrier under oxygen-limited conditions, where sucrose is present in excess (Table 2).

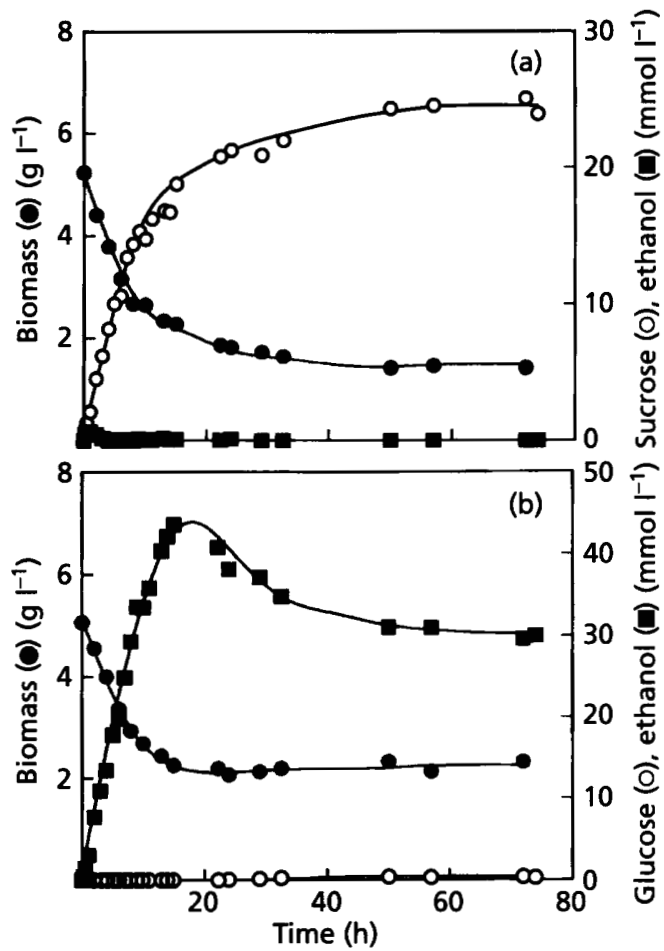

Fig. 2. Transient responses of $D$. yamadae CBS 7035 to oxygen limitation. At $t_{0}$ an aerobic sucrose-limited (a) or glucoselimited (b) chemostat culture $\left(D=0 \cdot 10 \mathrm{~h}^{-1}\right)$ was switched to oxygen-limited growth conditions. Transient-state experiments were performed in three independent chemostat cultures with essentially the same results. The figure shows data from a single experiment.

Indeed, sucrose-dependent alkalinization rates of washed cell suspensions harvested from oxygen-limited cultures were much lower than those of suspensions from aerobic cultures (Fig. 1a).

Although the above results clearly indicate the presence of a sucrose-proton symport mechanism in D. yamadae, the possibility that sucrose is also taken up by facilitated diffusion cannot be excluded.

\section{Transient responses of $\boldsymbol{D}$. yamadae to oxygen limitation}

If, as suggested above, sucrose metabolism can be regulated at the level of disaccharide uptake, the high capacity of the sucrose-proton symporter should be reduced during a switch from aerobic to oxygen-limited conditions. In practice, this can be achieved in two ways: by a cessation of the synthesis of new carrier molecules or by inactivation of the carrier molecules already present in the cell. In the former case, the transport capacity of the cells will only decrease slowly due to turn-over of the carrier molecules and dilution by growth. This may lead to a transient accumulation of ethanol, as observed in the case of maltose utilization by $C$. utilis (Kaliterna et al., 1995), even though ethanol is not detected in steady-state oxygen-limited cultures. In contrast, regulation of the 

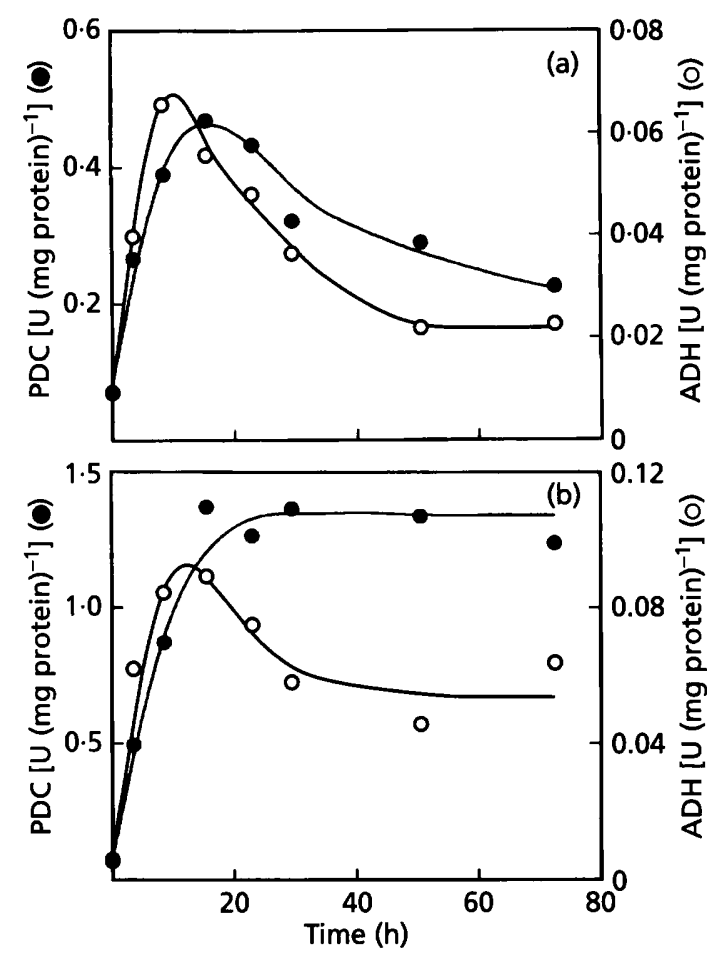

Fig. 3. Activities of alcohol dehydrogenase (ADH) and pyruvate decarboxylase (PDC) in D. yamadae CBS 7035 during a switch from aerobic to oxygen-limited conditions. At $t_{0}$ an aerobic, sucrose-limited (a) or glucose-limited (b) chemostat culture $\left(D=0.10 \mathrm{~h}^{-1}\right)$ was switched to oxygen-limited conditions.

activity of existing enzymes can occur over a time-scale of minutes (Holzer, 1976).

When an aerobic, sucrose-limited culture of D. yamadae was switched to oxygen-limited conditions, very low concentrations of ethanol $(<2 \mathrm{mM}$ ) were observed only during the first hours after the switch to oxygen-limited conditions (Fig. 2a) and sucrose started to accumulate in the culture within the first hour. From $5 \mathrm{~h}$ after the switch, neither ethanol nor other fermentation products were detected (Fig. 2a). When, as a control experiment, a glucose-limited chemostat culture was switched to oxygen limitation, this resulted in a rapid accumulation of ethanol and other fermentation products, without substantial accumulation of glucose (Fig. 2b).

\section{Activities of key fermentative enzymes}

Down-regulation of the key fermentative enzymes pyruvate decarboxylase and alcohol dehydrogenase has been suggested to contribute to the absence of alcoholic fermentation in yeasts that exhibit a Kluyver effect (Sims et al., 1991; Sims \& Barnett, 1991; Barnett, 1992). To investigate whether this might also hold for D. yamadae, activities of these enzymes were followed during switch experiments from aerobic to oxygen-limited conditions.

Both pyruvate decarboxylase and alcohol dehydrogenase were clearly induced under oxygen-limited conditions,
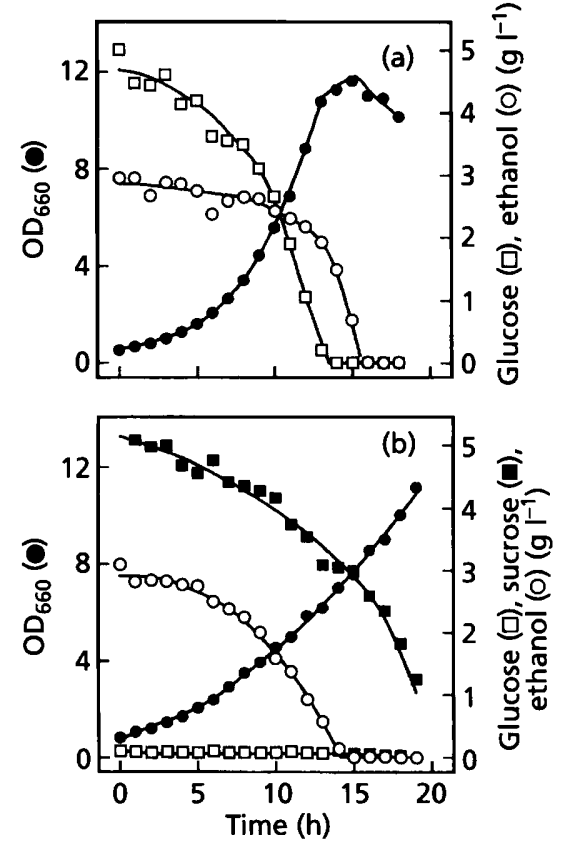

Fig. 4. Growth and substrate consumption of $D$. yamadae CBS 7035 in aerobic, $\mathrm{pH}$-controlled batch cultures on mixtures of (a) glucose and ethanol and (b) sucrose and ethanol. The media contained $5.0 \mathrm{~g}$ sugar $\mathrm{I}^{-1}$ and $4.0 \mathrm{~g}$ ethanol $\mathrm{I}^{-1}$.

activities being 10-20-fold higher than in the corresponding aerobic cultures (Fig. 3). In comparison with other facultatively fermentative yeasts, $D$. yamadae exhibited very low activities of alcohol dehydrogenase. Although even lower than in glucose-grown cultures, significant activities of alcohol dehydrogenase were detected in extracts from sucrose-grown, oxygen-limited cultures (Fig. 3).

Pyruvate decarboxylase was also present at significant activities in extracts from sucrose-grown, oxygen-limited cultures (Fig. 3). Therefore, the complete absence of alcoholic fermentation cannot be explained from a repression of the two fermentative key enzymes.

\section{Growth on mixtures of sucrose and ethanol}

It has recently been reported that in C. utilis and Debaryomyces castellii, two yeasts that exhibit the Kluyver effect for maltose and lactose, respectively, ethanol is preferred over the disaccharide during growth on mixtures of the two substrates. In contrast, preferential utilization of ethanol was not observed during growth of these yeasts on mixtures of ethanol and fermentable sugars (Weusthuis et al., 1994b).

To test whether preferential utilization of ethanol over sucrose occurred in D. yamadae, the yeast was cultivated in a $\mathrm{pH}$-controlled, aerobic fermenter with ethanol $\left(4 \mathrm{~g} \mathrm{l}^{-1}\right)$ and sucrose $\left(5 \mathrm{~g} \mathrm{l}^{-1}\right)$ as carbon sources. As a control, growth on a mixture of ethanol and glucose was studied. In contrast to the diauxic growth patterns observed with 
C. utilis and D. castellii, D. yamadae did not exhibit a preference for ethanol over the disaccharide. Instead, both glucose and sucrose were used simultaneously with ethanol (Fig. 4). The absence of extracellular glucose during batch cultivation on sucrose is consistent with the intracellular localization of the sucrose-hydrolysing enzyme.

\section{DISCUSSION}

A screening of eight yeast species that had previously been reported to exhibit a Kluyver effect for sucrose (Table 1) confirmed earlier reports (van Dijken et al., 1986; Barnett, 1992) about the insensitivity of the Durham-tube method, a procedure commonly used in yeast taxonomy to assess fermentation of carbohydrates. Therefore, the use of such tests may have led to an overestimation of the abundance of the Kluyver effect among facultatively fermentative yeasts. Nevertheless, three of the eight strains tested exhibited a clear Kluyver effect when ethanol formation was assayed by a sensitive enzymic method (Table 1).

The occurrence of a Kluyver effect for sucrose in $D$. yamadae was confirmed by studies in oxygen-limited chemostat cultures. During oxygen-limited growth on sucrose, no fermentation products were detected. In contrast, glucose-grown, oxygen-limited chemostat cultures produced substantial amounts of ethanol, glycerol and arabitol (Table 2). In steady-state oxygenlimited cultures, the molar ratio of ethanol to glycerol was approximately 3 . Thus, the relative amount of glycerol produced by $D$. yamadae is two- to three-fold higher than the maximum ratios observed in steady-state oxygenlimited chemostat cultures of $S$. cerevisiae and $C$. utilis (Weusthuis et al., 1994a). This high glycerol production may be related to the low alcohol dehydrogenase activities in this yeast (Fig. 3), which are one to two orders of magnitude lower than those in $S$. cerevisiae and $C$. utilis grown under comparable conditions (Weusthuis et al., 1994a; Kaliterna et al., 1995). Increased formation of glycerol has also been observed in S. cerevisiae mutants with an impaired synthesis of alcohol dehydrogenase (Johansson \& Sjöström, 1984).

The results presented in this paper demonstrate that sucrose metabolism in D. yamadae is initiated by its uptake across the plasma membrane, followed by intracellular hydrolysis, catalysed by an $\alpha$-glucosidase. This observation is consistent with the prediction made by Sims \& Barnett (1978) and Barnett (1981). It implies that, theoretically, the glycolytic flux during growth on sucrose can be controlled at the level of disaccharide uptake, as has been proposed for other yeasts that exhibit a Kluyver effect (Barnett \& Sims 1982; Kaliterna et al., 1995). Several observations presented here indicate that occurrence of the Kluyver effect, i.e. the adaptation of the glycolytic flux to the cells' respiratory activity, is indeed probably regulated at the level of sucrose uptake.

(i) The activity of the sucrose-hydrolysing enzyme is not repressed in oxygen-limited, sucrose-grown cultures.
Therefore, if down-regulation of the glycolytic flux were to occur at a reaction beyond sucrose uptake, accumulation of glucose and/or fructose would be expected after a switch to oxygen-limited conditions, rather than the observed accumulation of sucrose (Fig. 2).

(ii) Alkalinization studies suggest a decrease of the capacity of the sucrose-proton symporter after a switch to oxygen-limited conditions (Fig. 1). This observation should be interpreted with caution, since alkalinization is the net result of proton uptake by the sucrose carrier and acidification caused by the simultaneous action of the plasma membrane ATPase complex. In theory, the low alkalinization rates in oxygen-limited cultures could therefore also be due to an increased activity of the plasma-membrane ATPase under oxygen-limited conditions.

(iii) The Kluyver effect for sucrose in D. yamadae not only encompasses the absence of alcoholic fermentation, but also absence of glycerol and arabitol, products that branch off from the main glycolytic pathway (Table 2).

It should be stressed that intracellular hydrolysis of sucrose in a yeast does not necessarily imply the occurrence of a Kluyver effect. For example, sucrose metabolism in $H$. polymorpha is initiated by uptake via a proton-symport system and followed by intracellular hydrolysis by an $\alpha$-glucosidase (J. Kaliterna, unpublished results). Nevertheless, this yeast ferments sucrose under oxygen-limited conditions (Table 1).

The rapid accumulation of sucrose after a switch to oxygen-limited conditions (Fig. 2) suggests that in $D$. yamadae, regulation of sucrose-uptake capacity occurs at the level of enzyme activity. This contrasts with recent observations on the Kluyver effect for maltose in C. utilis, where a relatively slow decrease of the maltosemetabolizing activity appeared to be regulated at the level of enzyme synthesis (Kaliterna et al., 1995).

In contrast to observations made with $C$. utilis and $D$. castellii (Weusthuis et al., 1994b), the Kluyver effect for sucrose in $D$. yamadae was not associated with a preferential use of ethanol during growth on mixtures with the disaccharide (Fig. 4). This makes it unlikely that ethanol is a key effector molecule involved in the regulation of sucrose uptake in this yeast. Further work is needed to identify the kinetics, molecular mechanism and signals involved in down-regulation of sucrose uptake in D. yamadae after a switch to oxygen limitation.

Sucrose -proton symport has been extensively studied in plant cells (for a recent review see Bush, 1993). Comparatively little is known about uptake of sucrose by yeasts, although proton-symport systems have been demonstrated in some, including Candida albicans (Williamson et al., 1993) and S. cerevisiae (Santos et al., 1982). In the latter yeast, a sucrose-proton symport mechanism is present in addition to the well-characterized extracytosolic invertase. The apparent $K_{\mathrm{m}}$ for sucrose of $D$. yamadae estimated from alkalinization studies $\left(0.2 \mathrm{mmol} \mathrm{l}^{-1}\right.$; Fig. 1b) is lower than the value reported for $S$. cerevisiae and plant sucrose transporters (apparent 
$K_{\mathrm{m}}$ values of 6 and $1 \mathrm{mM}$, respectively; Santos et al., 1982; Bush, 1993), but is much higher than that of the C.albicans sucrose-proton symporter $\left(K_{\mathrm{m}}=7 \mu \mathrm{mol} \mathrm{l}^{-1}\right.$; Williamson et al., 1993). A further characterization of sucrose-proton symport in yeasts and a detailed comparison with the proton symporters of plants requires the use of isolated plasma-membrane vesicles. In view of its very high sucrose-proton symport activities in aerobic, sucroselimited chemostat cultures, $D$. yamadae seems an excellent model organism for such studies.

\section{ACKNOWLEDGEMENTS}

We are indebted to Dr James A. Barnett for valuable background information on sucrose transport in yeasts. We gratefully acknowledge the support of Pliva d.d., Zagreb, Croatia, to J. Kaliterna and a grant to J. I. Castrillo by the Department of Education, University and Research of the Basque Government, Autonomous Community of the Basque Country, Spain.

\section{REFERENCES}

Barnett, J. A. (1981). The utilization of disaccharides and some other sugars by yeasts. Adv Carbobydr Chem Biochem 39, 347-404.

Barnett, J. A. (1992). Some controls on oligosaccharide utilization by yeasts: the physiological basis of the Kluyver effect. FEMS Microbiol Lett 100, 371-378.

Barnett, J. A. \& Sims, A. P. (1982). The requirement of oxygen for the active transport of sugars into yeasts. $J$ Gen Microbiol 128, 2303-2312.

Barnett, J. A., Payne, R. W. \& Yarrow, D. (1990). Yeasts: Characteristics and Identification, 2nd edn. Cambridge: Cambridge University Press.

Bush, D. R. (1993). Proton-coupled sugar and amino acid transporters in plants. Annu Rev Plant Physiol Plant Mol Biol 44, 513-542.

Carvalho-Silva, M. \& Spencer-Martins, I. (1990). Modes of lactose uptake in the yeast species Kluyveromyces marxianus. Antonie Leeuwenboek 57, 77-81.

Dickson, R. C. \& Barr, K. (1983). Characterization of lactose transport in Kluyveromyces lactis. J Bacteriol 154, 1245-1251.

van Dijken, J. P., van Den Bosch, E., Hermans, J. J., Rodrigues de Miranda, L. \& Scheffers, W. A. (1986). Alcoholic fermentation by 'non-fermentative' yeasts. Yeast 2, 123-127.

Holzer, H. (1976). Catabolite inactivation in yeast. Trends Biochem Sci 1, 178-181.

Johansson, M. \& Sjöström, J. E. (1984). Enhanced production of glycerol in an alcohol dehydrogenase (ADH1) deficient mutant of Saccharomyces cerevisiae. Biotechnol Lett 6, 49-54.

Kaliterna, J., Weusthuis, R. A., Castrillo, J. I., van Dijken, J. P. \& Pronk, J. T. (1995). Transient responses of Candida utilis to oxygen limitation: regulation of the Kluyver effect for maltose. Yeast 11, (in press).
Noorman, H. J., Baksteen, J., Heijnen, J. J. \& Luyben, K. Ch. A. M. (1991). The bioreactor overflow device: an undesired selective separator in continuous cultures? J Gen Microbiol 137, 2171-2177.

Postma, E., Verduyn, C., Scheffers, W. A. \& van Dijken, J.P. (1989). Enzymic analysis of the Crabtree effect in glucose-limited chemostat cultures of Saccharomyces cerevisiae. Appl Environ Microbiol 55, 159-165.

Santos, E., Rodriguez, L., Elorza, M. V. \& Sentandreu, R. (1982). Uptake of sucrose by Saccharomyces cerevisiae. Arch Biochem Biophys 216, 652-660.

Schulz, B. \& Hofer, M. (1986). Utilization of lactose in nonrespiring cells of the yeast Debaryomyces polymorphus. Arch Microbiol 145, 367-371.

Serrano, R. (1977). Energy requirements for maltose transport in yeast. Eur J Biochem 80, 97-102.

Sims, A. P. \& Barnett, J. A. (1978). The requirement of oxygen for the utilization of maltose, cellobiose and D-galactose by certain anaerobically fermenting yeasts (Kluyver effect). $J$ Gen Microbiol 106, 277-288.

Sims, A. P. \& Barnett, J. A. (1991). Levels of activity of enzymes involved in anaerobic utilization of sugars by six yeast species: observations towards understanding the Kluyver effect. FEMS Microbiol Lett 77, 295-298.

Sims, A. P., Stăhlbrand, H. \& Barnett, J. A. (1991). The role of pyruvate decarboxylase in the Kluyver effect in the food yeast, Candida utilis. Yeast 7, 479-487.

van Urk, H., Schipper, D., Breedveld, G. J., Mak, P. R., Scheffers, W. A. \& van Dijken, J.P. (1989). Localization and kinetics of pyruvate metabolizing enzymes in Saccharomyces cerevisiae CBS 8066 and Candida utilis CBS 621. Biocbim Biopbys Acta 992, 78-86.

Van Leeuwen, C. C. M., Weusthuis, R. A., Postma, E., van den Broek, P. J. A. \& van Dijken, J. P. (1992). Maltose/proton cotransport in Saccharomyces cerevisiae. Comparative study with cells and plasma membrane vesicles. Biochem J 284, 441-445.

Weusthuis, R. A., Adams, H., Scheffers, W. A. \& van Dijken, J. P. (1993). Energetics and kinetics of maltose transport in Saccharomyces cerevisiae: a continuous culture study. Appl Environ Microbiol 59, 3102-3109.

Weusthuis, R. A., Visser, W., Pronk, J. T., Scheffers, W. A. \& van Dijken, J. P. (1994a). Effects of oxygen limitation on sugar metabolism in yeasts: a continuous-culture study of the Kluyver effect. Microbiology 140, 703-715.

Weusthuis, R. A., Luttik, M. A. H., Scheffers, W. A., van Dijken, J. P. \& Pronk, J. T. (1994b). Is the Kluyver effect caused by product inhibition? Microbiology 140, 1723-1729.

Williamson, P. R., Huber, M. A. \& Bennett, J. E. (1993). Role of maltase in the utilization of sucrose by Candida albicans. Biochem $J$ 291, 765-771.

Received 13 January 1995; revised 27 March 1995; accepted 10 April 1995. 